\title{
Brain endothelial cells-derived exosomes induce neuroplasticity in rats with ischemia/reperfusion injury
}

\section{Author information}

Beiyao Gao ${ }^{1} \uparrow$ Shaoting Zhou ${ }^{2} \uparrow$, Chengcheng Sun ${ }^{3}$, Dandan Cheng ${ }^{1}$, Ye Zhang ${ }^{3}$, Xutong Li ${ }^{2}$, Li Zhang ${ }^{1}$, Jing $\mathrm{Zhao}^{2 *}$,Dongsheng $\mathrm{Xu}^{456 *}$, Yulong Bai ${ }^{1 *}$

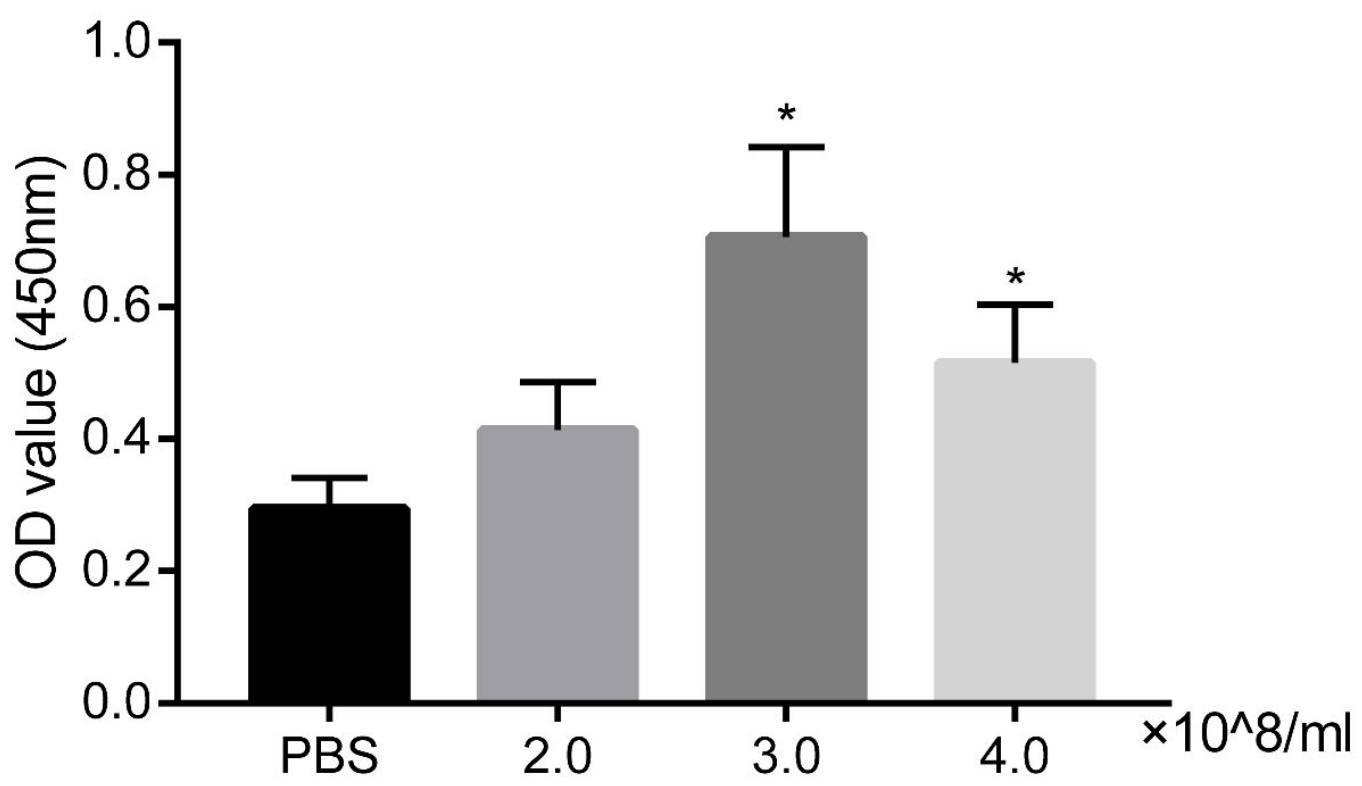

Figure S1 The effect of exosomes on PC12 cells proliferation at different concentrations

PC12 cells were treated with PBS or EC-Exo at $2.01 \times 10^{\wedge} 8,3.0 \times 10^{\wedge} 8$ and $4.0 \times 10^{\wedge} 8$ particles/ml after 72 hours of incubation, the CCK8 test was performed. Compared with the control group, the $O D$ value at $3.0 \times 10^{\wedge} 8$ and $4.0 \times 10^{\wedge} 8$ in the exosomes group were significantly increased $(P<0.01)$. Compared with the $3.0 \times 10^{\wedge} 8$ exosomes group, the 4.0 $\mathrm{x} 10^{\wedge} 8$ particles $/ \mathrm{ml}$ exosomes had no significant difference in the proliferation of PC12 cells. 